\title{
La gestión integral del riesgo en territorios urbanos
}

\author{
Maritza Duque Gutiérrez ${ }^{1}$, Jaime Quintero Olaya ${ }^{2}$ \\ 1. Ingeniera Agrónoma. MSc. Canditada a PhD en Conservación del Medio Natural-UNIA. \\ Secretaria Técnica del Sistema Integrado de Gestión Ambiental-U.D.C.A \\ 2. Ingeniero Civil. Especialista en Patología de la Construcción - Gestor del Riesgo.
}

Correspondencia: maduque@udca.edu.co, jaimearchivos $2 @$ gmail.com

Recibido: 28/05/2013 Aceptado: 28/06/2013

\section{Resumen}

$\mathrm{Al}$ igual que en el resto del planeta, Colombia está sufriendo un importante flujo migratorio desde el medio rural a las ciudades, aspecto que se ha intensificado por el conflicto armado. Este incremento de la superficie urbana está generando importantes transformaciones en sus territorios adyacentes, que mantiene ecosistemas y comunidades biológicas de gran valor ecológico. Aunado a esto, la población se enfrenta a situaciones de riesgo por fenómenos de remoción en masa, inundación, entre otros, ya que están ubicadas en zonas de ladera o cercanas a fuentes de agua.

Aunque se están realizando importantes esfuerzos por reducir la demanda de recursos y sus residuos, sigue incrementándose la degradación de los ecosistemas colindantes con la consiguiente pérdida de biodiversidad y la alta probabilidad de materialización de situaciones de riesgo para la población. Este artículo busca describir los parámetros de una línea de investigación que conceptúe a la ciudad como un socioecosistema, como una alternativa que permite hacer gestión del riesgo.

Palabras clave: ecosistemas, gestión de riesgo, planificación territorial, socioecosistema.

\section{Integrated risk management in urban areas}

\begin{abstract}
As in the rest of the planet, Colombia is undergoing an important migratory flow from rural areas to the cities, an aspect that has been intensified by the armed conflict. This growth in the urban area is generating significant transformations in their adjacent territories, which maintain ecosystems and biological communities of great ecological value. Also, the population is facing situations of risk by phenomena like mass removal and floods, among others, as they are located in hilly areas or close to water sources.

Although major efforts are being made to reduce the demand for resources and their residues, the degradation of bordering ecosystems continues to increase with the consequent loss of biodiversity and the high probability of materialization of risk situations for the population. This article seeks to describe the parameters of a line of research that defines the city as a socio-ecosystem, as an alternative that allows you to make risk management.
\end{abstract}

Keywords: ecosystems, risk management, planning, socio-ecosystem. 


\section{Introducción}

Los servicios de los ecosistemas son los beneficios que reciben las personas de la naturaleza (1). Para su comprensión se dividen en servicios de abastecimiento como los suministros directos por parte de la naturaleza (el agua y el alimento); los servicios de regulación, llamados así a los procesos metabólicos necesarios (como la regulación hídrica y la fertilidad); y los servicios culturales que son los servicios resultante de la relación estrecha humanos-naturaleza (como la identidad y la educación ambiental) (2-5).

Estos servicios han representado una importante disminución debido a la presión de las acciones de los humanos sobre los ecosistemas, lo que también se conoce como impulsores directos de cambio, que en su orden de importancia son los cambios en los usos del suelo, el cambio climático, la contaminación, la introducción de especies invasoras, los cambios en los ciclos biogeoquímicos y la sobreexplotación (6).

Estas alteraciones han generado que algunos científicos definan este período como el Antropoceno (7), caracterizado por los cambios sin precedentes que ocasionan las actividades humanas sobre el metabolismo y dinámicas del planeta (8). Los procesos biofísicos se han visto alterados poniendo en peligro el bienestar de las personas que viven directamente del flujo de servicios de los ecosistemas (9).

Lo anterior se intensifica cuando más personas demandan estos servicios. En solo 2 siglos la población se ha incrementado en siete veces y mientras que en 1900 solo el 10\% de la población mundial vivía en ciudades, ese porcentaje actualmente es de más del $50 \%$ y aumentará en los próximos 50 años (10). Además se presenta un aumento de las ciudades que sobrepasan los 10 millones de habitantes. Es así como la urbanización y la re-densificación interna en las ciudades y las necesidades de nuevos escenarios para habitar se han convertido en uno de los procesos de mayor importancia que promueve el Cambio Global (11).
Este tema ha sido de tal relevancia que ya en la Cumbre de la Tierra en 1992 se ponía de manifiesto que el futuro de la sostenibilidad del planeta se decidiría en las grandes ciudades. Incluso en Río +20, el tema de ciudades sostenibles hizo parte de las siete áreas que requiere atención prioritaria. Consecuentemente, en la Conferencia "Planeta Bajo Presión 2012” (12), en el reporte especial sobre el manejo del riesgo por eventos extremos y desastres para avanzar en la adaptación al cambio climático, se concluye que el desafío hacia el futuro debe tener una dimensión enfocada al desarrollo de conocimiento basado en el empoderamiento de decisiones, incluso para aquellas situaciones en las que los niveles de incertidumbre son muy altos.

Lo anterior puede entenderse como la generación del conocimiento en el marco de la Gestión Integral del Riesgo de que trata la Normatividad Colombiana (13) asociada sobre las amenazas, vulnerabilidades y su posibilidad de materialización sobre la población y sus bienes, la infraestructura, el ambiente, la economía y el desarrollo de la ciudad de Bogotá, siendo esto una gran herramienta para la toma de decisiones a diferentes escalas sociales.

Ya desde 1960 comenzaban a hacerse las primeras aproximaciones a la ciudad sostenible al generar un análisis desde su metabolismo (14). Actualmente se presenta la moda de las Smart cities dando a la tecnología la responsabilidad para resolver los problemas de la ciudad (15) presentándose, en ocasiones, que las ciudades que ocupan los primeros puestos con la etiqueta de "inteligentes", ocupan también los primeros en huella ecológica.

El resultado es que cada vez más nos alejamos de crear una ciudad que se prepara frente a los riesgos, por la visión compartimentada que se ha tenido: los biólogos se encargan de estudiar la avifauna urbana, los antropólogos de tratar explicar los fenómenos sociales urbanos, los ingenieros brindan soluciones estructurales requeridas de acuerdo al 
análisis técnico del territorio el cual se enmarca en un proceso de ocupación carente de planificación, generando así una sectorización del conocimiento sobre la ciudad.

Se entiende entonces, que es necesario crear un marco integral e integrador para gestionar las ciudades como un todo y no en un territorio subdividido, y así romper con las dualidades centroperiferia, urbano-rural y crear ciudades capaces de gestionar y reducir los diferentes eventos a los que está sometida. Es decir, hacer gestión integral del riesgo enfocada a la prevención sobre las diferentes condiciones presentadas en las amenazas y las condiciones de vulnerabilidad acordes con los niveles de exposición existentes, evitando con ello la materialización del riesgo, mediante el desarrollo de medidas de reducción del mismo. Con estas condiciones y un fuerte nivel de desarrollo del conocimiento, se logrará brindar estrategias de fortalecimiento de la capacidad de respuesta tanto del individuo como de lo construido $(13,16,17)$ y que son componentes fundamentales del socioecosistema; elevando con ello los niveles de resiliencia para el bienestar humano (18).

Aunque se han realizado aproximaciones al respecto, faltan lineamientos y apropiación del riesgo de forma integrada, que permitan analizar a la ciudad como un sistema complejo adaptativo, lo que corresponde a su conceptualización como socioecosistema, proveyendo un marco para considerar las dimensiones ecológica y social, así como sus interrelaciones (19, 20). Por tanto el objetivo principal de este artículo es generar una primera aproximación conceptual de la ciudad de Bogotá como un socioecosistema que aumenta la apropiación del riesgo y su gestión.

\section{La ciudad de Bogotá se prepara para afrontar y gestionar los riesgos}

Bogotá es la ciudad más poblada de Colombia, con cerca del $20 \%$ del total de su población. Actualmente es el mayor centro urbano y cuenta con
7.363.782 habitantes y 7.347.795 en el área metropolitana (21) con una densidad poblacional de cerca de 4.146 habitantes $/ \mathrm{km}^{2}$, ocupando así el puesto 37 a nivel mundial y el 5 a nivel Latinoamérica. Solamente el $17 \%$ de su superficie urbana recoge cerca del $80 \%$ de la población (21).

Desde su fundación en 1536 hasta mediados del siglo XIX, la ciudad no presentó mayores cambios. Solamente después de 1850 empezó a experimentar un pequeño crecimiento, que ya fue notorio a finales de siglo XIX. Hacia 1920, tenía las mismas construcciones que 100 ańos atrás, pero cinco veces más su población y los problemas de la estructura urbana se hicieron evidentes $(22,23)$. En las décadas de 1940, 1960 y 1970 se observaron los incrementos más significativos en la población (Figura 1) que fueron desproporcionados frente a los recursos disponibles en la ciudad, los cuales se manifestaron principalmente en la cobertura del transporte, los servicios públicos y la oferta de empleo $(24,25)$.

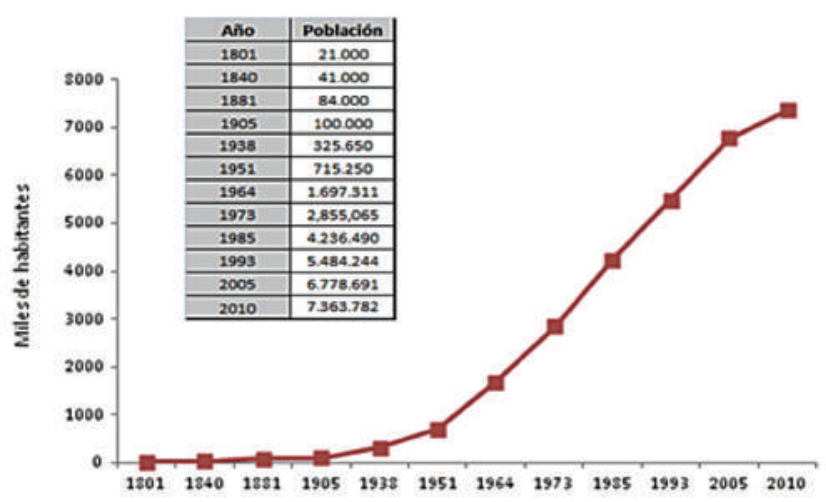

Figura 1. Crecimiento poblacional de la ciudad desde 1801 hasta 2010. Se observa un crecimiento de la población rápido y exponencial a partir de 1940 .

Bogotá pasó de tener una población en 1905 de 100.000 habitantes a 6.778.691 habitantes en 2005, convirtiéndose en la ciudad más poblada de Colombia, con una representación del 14,3\% del total de población del país (21), ubicada en un 99.7\% en suelo urbano. 
Este crecimiento acelerado se realizó de manera desorganizada, sin ningún tipo de control sobre la utilización del suelo, se apropiaron suelos de preservación ambiental, humedales, corredores ecológicos, zonas de ladera en donde se emplazaron asentamientos humanos no planificados en construcciones carentes de un asesoramiento técnico, la implementación de procesos de explotación minera (canteras, chircales, etc.) sin las medidas respectivas de recuperación y estabilización posteriores, generando nuevos procesos de inestabilidad ante fenómenos de remoción en masa en el territorio llevando consigo el deterioro de las condiciones ambientales del entorno (26).

Junto a bajas tasa de mortalidad y altas de fecundidad, los movimientos migratorios en Colombia, resultan ser un factor clave en el momento de explicar las dinámicas poblacionales de un territorio. Estas migraciones son el resultado de una ciudad que resulta ser atractiva como centro de oportunidades de desarrollo laboral o de formación académica y también por cuestiones de orden público, para huir de la violencia de los grupos armados. Bogotá se ha convertido en el destino final para el cerca del 32\% de la población migrante del país (21).

Esto genera, en primera instancia, un deterioro del capital social seguido del aumento de presión sobre los ecosistemas colindantes al aumentar el flujo de ecoservicios para satisfacer las necesidades de la población urbana (27). Lo anterior significa que cada vez se demandan más materiales, energía y agua, con la subsecuente generación de residuos al entorno sin previo tratamiento (metabolismo lineal) $(28,29)$.

Surge entonces la necesidad de dar una nueva mirada a Bogotá, no solo como la ciudad que vive de los servicios que se generan en el entorno, sino como una ciudad capaz de generar servicios y que garantice el bienestar de sus habitantes. Si bien es cierto que la implementación de tecnologías verdes y el uso de estrategias para minimizar impactos (metabolismo circular) ha sido un gran avance
(30), es necesario que Bogotá atienda las problemáticas sociales que le aquejan desde una perspectiva más holística en su conceptualización como socioecosistema.

\section{¿Cómo la ciudad de Bogotá debe hacer gestión del riesgo?}

Dentro del análisis complejo que debe abordar la comprensión y gestión de las ciudades y las dinámicas poblacionales que se han presentado, se realiza, en primera instancia, una mirada relevante a la cuestión social, conformada por la dinámica social y la gobernanza (31) (Figura 2). Hasta muy reciente la planificación urbana se centraba en la creación de lugares atractivos, eficientes y equitativos, pero esto ha estado lejos de crear un espacio inclusivo de todos los actores que se convierten en la parte invisible que construye ciudad y generan una planificación urbana de "abajo a arriba" (32).

Este desconocimiento ha generado un fracaso por la incapacidad para comprender cómo funcionan las ciudades, ya que las reacciones políticas e ideológicas se han mantenido en la sombra. La ciudad no es más que la sumatoria de sus partes y de ver solamente algunas de sus partes nace el desconocimiento en cuanto a su naturaleza $(33,47)$. La diversidad y variedad cultural de las ciudades son su señal de identidad, viéndose plenamente afectadas por la urbanización contemporánea $(35,36)$.

Pensar de abajo hacia arriba es una alternativa de integración. El modelo en el que se ha basado la ciudad es estático, a primera vista parece serlo en su estado físico (estructuras, edificios, calles), pero las dinámicas socioeconómicas que están dentro mantienen un flujo constante, lo que lleva a que una ciudad puede mantenerse físicamente en el tiempo pero su funcionalidad y comportamiento varían. Un mismo patrón espacial puede surgir de fenómenos diferentes, considerando que es la parte invisible (la social) cuyas acciones de individuos y organizaciones, construyen ciudad en una escala más significativa que la planificación urbana $(37,38)$. 
En este contexto, el componente social es fundamental, pero no es general en la ciudad, es necesaria también su comprensión ecológica, que en el espacio urbano se entiende como el urbanismo ecológico y el metabolismo. Es así como esta propuesta integra 4 esferas: las dinámicas sociales, la gobernanza adaptativa, el urbanismo ecológico y el metabolismo urbano como propuesta integral e integradora para generar bienestar humano (39) (Figura 2).

Para nuestro efecto hemos definido cada uno de los componentes:

- Dinámicas sociales: el estudio de temas relacionados a la población y el poblamiento (40).
- Gobernanza adaptativa: hace referencia a las estructuras institucionales y las organizaciones (40).

- Urbanismo ecológico: tiene como objetivo la preservación de valores geográficos y naturales, la gestión de la logística urbana (servicios urbanos como el agua, el gas y las telecomunicaciones), la movilidad, el espacio público y las tecnologías (41).

- Metabolismo circular: proceso lineal mediante el cual el humano urbano usa recursos y los retorna al medio de una forma de menor calidad (gases, vertidos, residuos sólidos) (41).

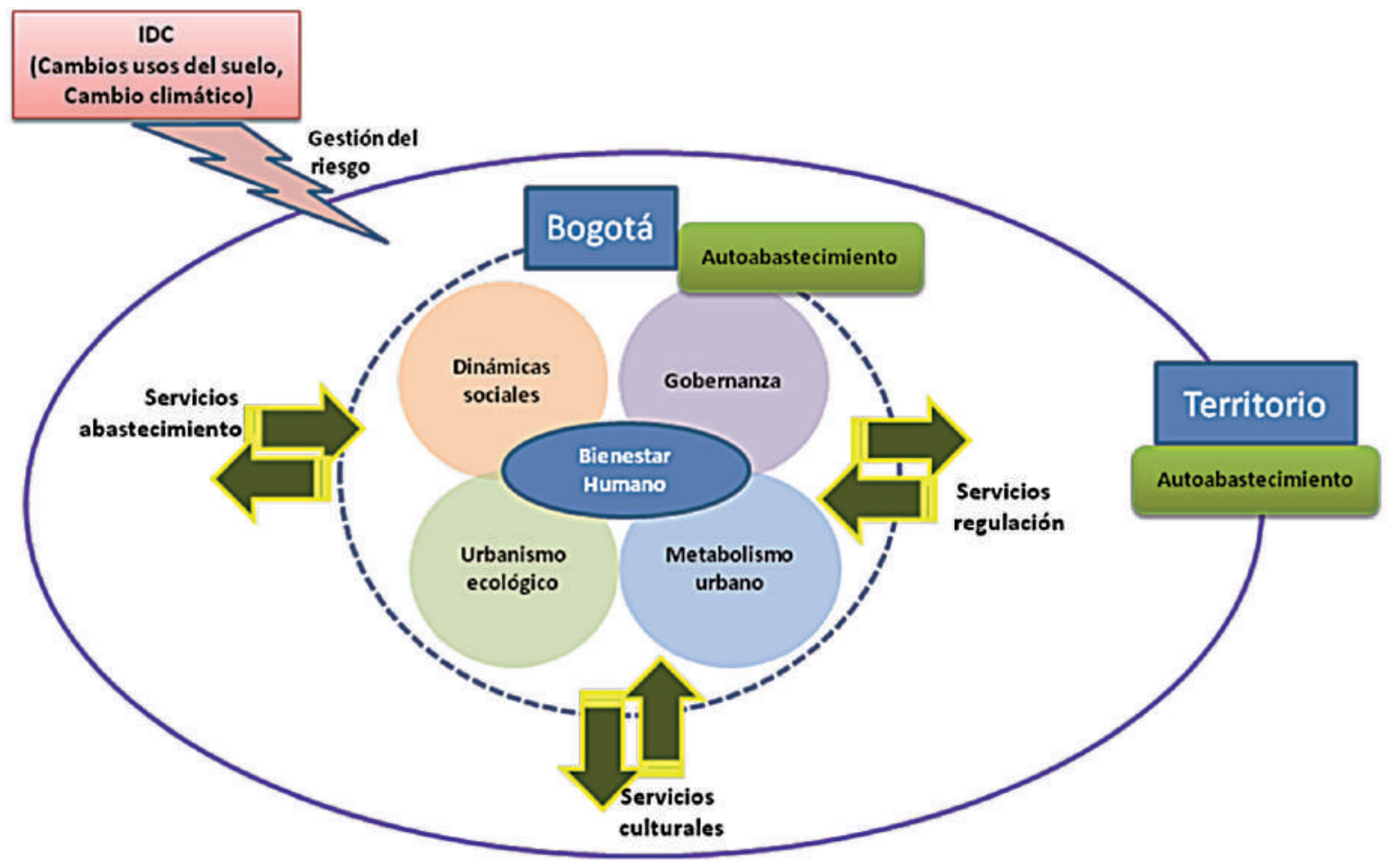

Figura 2. Marco conceptual para el análisis de Bogotá. En esta propuesta la ciudad se convierte en un ecosistema más del territorio con la capacidad, a través de sus áreas verdes, de generar servicios tanto para su autoabastecimiento como también a escala territorial. Para esto se entiende el fenómeno emergente de la interacción de las componentes sociales y ecológicas urbanas para generar bienestar humano urbano. Del hacer frente a los impulsores directos de cambio resulta la gestión del riesgo (Modificado de 39).

Consecuentemente se debe buscar una articulación de instrumentos de planificación y estrategias de intervención propias para cada uno de los territorios (acciones específicas para cada división y subdivisión territorial), ya que cada uno de estos tiene dinámicas no lineales, así como mecanismos sectoriales vinculantes para todos, garantizando una adecuada reducción y gestión del riesgo (42), para lograr el bienestar humano de todos los ciudadanos y ciudadanas. 


\section{Discusión}

Uno de los grandes retos de Bogotá como una ciudad que desde hace 25 años está cambiando cada vez más rápido, es hacer gestión del riesgo enfocado a la resiliencia urbana en un contexto territorial para alcanzar el bienestar humano, y más cuando es el mismo comportamiento humano el que está logrando transformaciones sin precedentes (43). Esto se ha manifestado cuando se observa que las interacciones naturaleza-sociedad se han visto desacopladas, generando la crisis que estamos viviendo día a día, que en su forma visible se muestra como una crisis financiera pero que está sustentada por una crisis social y ecológica, es decir, una crisis de la sociedad $(44,45)$.

Aun así, esta problemática se muestra como una ventana de oportunidades. Si las varias iniciativas que se han planteado y aplicado a Bogotá no han logrado detener esta crisis, por la estrategia compartimentada de ver a las sociedades y ecosistemas, es hora de generar una propuesta integral e integradora. Por esta razón, lo que representa la figura 2 se convierte en una respuesta para gestionar a Bogotá en el contexto de cambio global, en el que la ciudad solo puede entenderse en un contexto territorial (46) para hacer gestión del riesgo.

Una vez que se definieron los componentes que deben actuar de forma sinérgica en la ciudad (dinámicas sociales, gobernanza adaptiva, urbanismo ecológico y metabolismo urbano), se busca reducir la demanda de servicios por parte de la ciudad, convirtiéndose esta última en una generadora de servicios a través de sus áreas verdes: como la producción de alimentos en los huertos, la regulación climática a través del arbolado urbano o la identidad a través del espacio público.

Lo anterior debe ser resultado de una adecuada planificación regional y sectorial de Bogotá en su contexto como capital, con efectos vinculantes buscando bases solidas para la gestión del riesgo, incluyendo la creación de instrumentos financieros de desarrollo en el ámbito regional y la definición de un sistema de articulación y coordinación de la gestión integral del riesgo donde cada actor responsable desde su función, interactué para construir cuidad (42).

Como consecuencia inicial de la propuesta se espera resultados en la conservación de la red hídrica urbana como humedales y corredores ecológicos, definición y construcción de espacios públicos verdes urbanos, que incluyan la apropiación mediante la educación ambiental en el contexto urbano. Si bien la ciudad nunca podrá ser sostenible completamente, si puede reducir el impacto que actualmente está generando y puede convertirse en un espacio, que siendo heterotrófico, aumente su autoabastecimiento.

Finalmente, se sugiere que una de las metas de la planificación territorial y sectorial sea la construcción de un mapa dinámico de riesgos multicriterios, acorde con la condición actual de la ciudad y siendo un modelo cambiante en el tiempo; el cual esté incluido al socioecosistema que se busca consolidar, el que debe ser evaluado a través de indicadores de frecuencia-intensidad, que incluya los destinatarios de los servicios de ciudad y sus efectos de forma específica (sectorial), o de agrupación (destinatarios en un territorio) (42).

\section{Referencias}

1. De Groot, R., Wilson, M., \& Boumans, R. . A typology for the classification,. Ecological Economics. 2002; 41:393-408.

2. MA (Millennium Ecosystem Assessment). 2003. Ecosystems and human well-being: A framework for assessment. Island Press, Washington, D.C.

3. Hein L., Van Koopen K., de Groot R.S., Van Ierland E.C. 2006. Spatial.

4. Carpenter, S., Mooney, H., Agard, J., Capistrano, D., DeFries, R., Dietz, T., .. Whyte, A. . Science for managing ecosystem services: beyond the Millennium Ecosystem . Proceedings of the National Academy of Science. 2009; 106(5): 1305-1312.

5. Duque, M. Los servicios de los ecosistemas de Chocontá: un ejercicio de percepción con sus habitantes. NOVA, (2011) 9(15): 113-214.

6. Duarte Cm., Aabanades Jc., Agustìn S., Alonso S., Benito G., Ciscar Jc., Dachs J., Grimalt Jo., Lopez., Montes C., Pardo M., Rios Af., Simò R., y Vallardes F. Cambio global: impacto de la actividad humana sobre el sistema tierra. Consejo Superior de Investigaciones Científicas. Madrid. 2009.

7. Crutzen, P. Geology of mankind: the anthropocene. Nature. 2002; 415:23. 
8. Steffen, W., $\mathrm{A}^{\circ}$. Persson, L. Deutsch, M. Williams, J. Zalasiewicz, C. Folke, J. Rockstro"M, C. Crumley. 2011. The Anthropocene: From global change to planetary stewardship. Ambio. doi: 10.1007/s13280-011-0185-x.

9. MA (Millennium Ecosystem Assessment). Ecosystems and human well-being:Synthesis. Island Press, Washington, D.C. 2005.

10. Grimm, N; Faeth, S; Golubiewski, N; Redman, C; Wu, J; Bai, X; Briggs, J. 2008. Global Change ant the Ecology of cities. Science 319. 756-760 scales, stakeholders and the valuation of ecosystem services. Ecological Economics, 57: 209-228.

11. Terradas J., Franquesa T., Parès M., Chaparro L. 2011. Ecología Urbana. Revista Investigación y tecnología.

12. Planet under pressure Conference. Special Report on Managing the Risks of Extreme Events and Disasters to Advance Climate Change Adaptation (SREX). 2012.

13. PRESIDENCIA DE LA REPÚBLICA DE COLOMBIA. Ley 1523 de 2012. Política Nacional de Gestión del Riesgo de Desastres. Bogotá.

14. TERRADAS J. Ecología Urbana. Barcelona, Rubes Editorial, S. L. 2001.

15. Schaffers, H., Komninos, N., Pallot, M., Trousse, B., Nilsson, M., Oliveira, A. (2011). Smart Cities and the Future Internet: Towards Cooperation Frameworks for Innovation. In: J. Domingue et al. (Eds.), The Future Internet, Springer, pp 431-446.

16. ALCALDÍA MAYOR DE BOGOTÁ. Decreto 332 de 2004. Sistema para la Prevención y Atención de Emergencias en Bogotá. Bogotá.

17. ALCALDÍA MAYOR DE BOGOTÁ. Decreto 423 de 2006. Plan Distrital para la Prevención y Atención de Emergencias para Bogotá. Bogotá.

18. Montes, C., \& Sala, O. (2007). La Evaluación de los Ecosistemas del Milenio. Las relaciones entre el funcinamiento de los ecosistemas y el bienestar humano. Ecosistemas, 16 (3):137-147.

19. Ostrom, E. Crossing the great divide: coproduction, synergy and development. World Development. 1996; 24(6): 1073-1087.

20. Ostrom, E., 2009. A general framework for analyzing sustainability of social- ecological systems. Science 325 (5939), 419.

21. DANE. 2005. Censo General de Población 2005. Publicación digital en la página web: http://www.dane.gov.co/censo/

22. Zambrano, F. Historia de Bogotá. Siglo XX. Bogotá: Villegas Editores. 2007.

23. Maya T. 2010. Áreas residenciales y desarrollo urbano en Bogotá. Revista Urbanismos, No 2. Bogotá: Facultad de Artes, Universidad Nacional de Colombia. (Segundo semestre, 2004). 23-55.

24. DANE. (1985). DANE. Obtenido de Archivo Nacional de Datos: http://190.25.231.249/metadatos/index.php/catalog/148

25. DANE. (2006). Departamento Administrativo Nacional de Estadistica. Obtenido de PIB por ramas de actividad corrientes y constantes 1990-2005: http://www.dane.gov.co/index.php?option=com content\&view=article\&id=341\&Itemid=86\&lang=es

26. ALCALDÍA MAYOR DE BOGOTÁ. Lineamientos y Marco de Politica Distrital de Reasentamiento. Bogotá. 2008.

27. Grimm, N., Redman, C., Boone, C., Childers, D., Harlan, S., \& Turner II, B. (2013). Viewing the Urban Socio-ecological System Through a Sustainability Lens: Lessons and Prospects from the Central Arizona-Phoenix LTER Programme. En S. Singh, H. Haberl, V. Gaube, C. Grünbühel, P. Lisivieveci, J. Lutz, ... . M. Wildenberg, Long term socio-ecological research. Human-environment interactionsSpringer (págs. 217-246). New York: Springer.
28. Rueda, S. Ecología urbana. Barcelona: Beta Editorial. 1995.

29. Rueda, S. Barcelona, ciudad mediterránea, compacta y compleja. Una visión de futuro más sostenible. Barcelona: Ayuntamiento de Barcelona. 2002.

30. Rueda, S. El urbanismo ecológico: un nuevo urbanismo para abordar los retos de la sociedad actual. Obtenido de http://www. upv.es/contenidos/CAMUNISO/info/UrbanismoEcologicoSRueda.pdf. 2009.

31. Berkes, F., \& Folke, C. Linking social and ecological systems. Management practices and social mechanisms for building resilience. Cambridge: Cambridge University Press. 1998.

32. Batty M., Barros J., Junior S., 2004. Cities: continuity, transformation, and emergence. Working paper 72, CASA UCL, London.

33. Simon, H. A. The Architecture of Complexity, Proceedings of the American Philosophical Society. 1962; 106, 467-482.

34. Palacio, G. 2008. Historia ambiental de Bogotá y la Sabana, 1850-2005. Universidad Nacional de Colombia Sede Amazonía, IMANI. Leticia, Amazonas, Colombia.

35. Jacobs, J. The Death and Life of Great American Cities, Random House, New York. 1961.

36. Alberti, M., \& Marzluff, J. Ecological resilience in urban ecosystems: Linking urban patterns to human and ecological functions. Urban Ecosystems. 2004; 7: 241-265.

37. Colding, J. Ecological land-use complementation for building resilience in urban ecosystems. Landscape and Urban Planning. 2007; 81 (1-2): 46-55.

38. Colding, J., Lundberg, J., \& Folke, C. Incorporating green area user groups in urban ecosystem management. Ambio. 2006; 35(5): 237-44

39. Duque, M. Gestionando la ciudad de Bogotá como un socioecosistema. Una aproximación desde la Nueva Ecología Urbana. Trabajos en extenso I Congreso Internacional de Ecología Urbana. Buenos Aires. 2012

40. Resilience Alliance (2007), Urban Resilience: Research Prospectus a Resilience Alliance Initiative for Transmitting Urban Systems towards Sustainable Futures.

41. Rueda, S. Libro verde del medio ambiente urbano. Agencia de ecología Urbana de Barcelona. Barcelona. 2007.

42. Fleischhauer, M., Wanczura, S., \& Greiving, S. (2007). Planificación Territorial para la Gestión de Riesgos en Europa.

43. Duque, M. Gestionando el capital natural de la Sabana de Bogotá para el bienestar humano: una aproximación a su concepción como socioecosistema. Laboratorio e Socioecosistemas, Universidad Autónoma de Madrid. Madrid. 2010.

44. Constanza, R. Ecological Economics: the science and management of sustainability. New York: Columbia University Press. 1991.

45. Constanza, R., Waigner, L., Folke, C., \& Maler, K.. Modeling complex ecological economic systems: towards an evolutionary dynamic understanding of people and nature. BioScience. $1993,43: 545-555$.

46. Pickett, S., Cadenasso, M., Grove, C., Nilon, C., Pouyat, R., Zipperer, W., Constanza, R. Urban ecological systems: linking terrestrial ecological, physical, and socioeconomic components of metropolitan areas. Annual review of ecology and systematic. 2001; 32: 127-57. 